2. Bouton ME, Westbrook RF, Corcoran KA, Maren S. Contextual and temporal modulation of extinction: behavioral and biological mechanisms. Biol Psychiatry. 2006;60:352-60.

3. Denny CA, Kheirbek MA, Alba EL, Tanaka KF, Brachman RA, Laughman KB, et al. Hippocampal memory traces are differentially modulated by experience, time, and adult neurogenesis. Neuron. 2014;83:189-201.

4. Liu X, Ramirez S, Pang PT, Puryear CB, Govindarajan A, Deisseroth K, et al. Optogenetic stimulation of a hippocampal engram activates fear memory recall. Nature. 2012;484:381-385.
5. Lacagnina AF, Brockway ET, Crovetti CR, Shue F, McCarty MJ, Sattler KP, et al. Distinct hippocampal engrams control extinction and relapse of fear memory. Nat Neurosci. 2019;22:753-61.

6. Khalaf O, Resch S, Dixsaut L, Gorden V, Glauser L, Gräff J. Reactivation of recallinduced neurons contributes to remote fear memory attenuation. Science. 2018;360:1239-42.

\title{
The complexity of pharmacology of cannabidiol (CBD) and its implications in the treatment of brain disorders
}

\author{
Sonja Elsaid ${ }^{1,2}$ and Bernard Le Foll ${ }^{1,2,3}$ \\ Neuropsychopharmacology (2020) 45:229-230; https://doi.org/10.1038/s41386-019-0518-1
}

Cannabidiol (CBD) is one of the major cannabinoid constituents of the Cannabis plant. Recently, CBD has sparked the interest of medical researchers because of its more than 65 identified molecular targets. Of those, mostly studied in brain disorders are cannabinoid, $5 \mathrm{HT}_{1 \mathrm{~A}}$ receptors, G-protein receptor protein 55 (GPR55), transient receptor potential (TRP) channels, and cytochrome P450s [1]. Here we discuss possible mechanisms of actions of $C B D$ in several brain disorders.

The evidence suggests that the antiepileptic potential of CBD may be via its modulation of TRP (vanilloid 1 and TRPA), potassium channels, NMDA receptors, and more recently by the interaction with GPR55 to reduce neuronal excitability [1, 2]. Although in the US, CBD is currently prescribed as an adjuvant treatment for seizures in Lennox-Gastaut and Dravet syndromes, as well as tuberous sclerosis complex, it is still unknown if CBD's antiepileptic properties are due to its direct interaction with the molecular targets, or possibly through potentiating effects of antiepileptic treatments by modulation of cytochrome P450s [1, 3].

CBD exhibited anxiolytic properties by acting on the $5 \mathrm{HT}_{1 \mathrm{~A}}$ receptors in animal models [1]. Most recently, an in vitro study showed that CBD might also elicit anxiolytic effects by allosterically modulating $\mathrm{GABA}_{\mathrm{A}}$ receptors [4]. Human studies using $\mathrm{CBD}$ were limited to assessing the short-term effects of $C B D$ on social anxiety disorder (SAD) [1].

By the mechanism of action on the $C B 1$ receptor, $C B D$ attenuated behavioral responses to different forms of aversive memories in rodent PTSD models [5]. Although in human studies, CBD was associated with reduced PTSD symptomatology, the evidence is only limited to case studies, while possibly being confounded by the co-administration of other psychiatric treatments [6].

The antidepressant properties of $\mathrm{CBD}$ by activation of $5 \mathrm{HT}_{1 \mathrm{~A}}$ receptors were revealed in animal models of depression [1]. However, to date, CBD's effects on clinical depression have not been studied.
CBD has been proposed to have anti-psychotic effects by modulating dopamine D2, cannabinoid receptors, and TRPV1 channels; however, these mechanisms are somewhat speculative, given the lack of reproducibility of findings. In human studies, CBD produced conflicting evidence to either augment or improve the symptoms of schizophrenia [1].

The anti-addictive potential of CBD was demonstrated in animal models of cannabis, opioid, alcohol, methamphetamine, and cocaine use disorders. Although CBD's molecular pathways are still poorly understood, they may include neuronal excitability, $5 \mathrm{HT}_{1 \mathrm{~A}}$ receptors and possibly cannabinoid and opioid systems. In small-scale clinical trials, CBD reduced cigarette consumption and heroin cue-induced craving. The anecdotal evidence also shows the positive effects of CBD on reducing symptoms of cannabis and alcohol use disorders, yet these effects need further investigation in larger trials [1].

In summary, the complexity of CBD pharmacology is due to CBD's ability to interact with several molecular targets, making it a good candidate for further therapeutic investigation. Currently, in the US, CBD is only prescribed for treatment of childhood epilepsies, while other indications are still under exploration. To fully elucidate its true therapeutic potential in other brain disorders, CBD needs to be tested in larger-scale randomized, placebo-controlled trials.

\section{FUNDING AND DISCLOSURE}

BLF has received in-kind donation of cannabis products from Canopy Innovations Inc. and Aurora Cannabis and medication donation from Pfizer and Bioprojet. He was provided a coil for the TMS study from Brainsway. BLF will conduct research with funding obtained from Canopy Innovations Inc. and Aphria (through research grants handled by $\mathrm{CAMH}$ and University of Toronto), Bioprojet, ACS and Alkermes. BLF has received in kind donations of nabiximols from GW Pharma for studies funded by $\mathrm{CIHR}$ and $\mathrm{NIH}$. The authors declare no competing interests.

\footnotetext{
${ }^{1}$ Translational Addiction Research Laboratory, Centre for Addiction and Mental Health (CAMH), 33 Russell Street, T-700, Toronto, ON M5S 2S1, Canada; ${ }^{2}$ The Institute of Medical

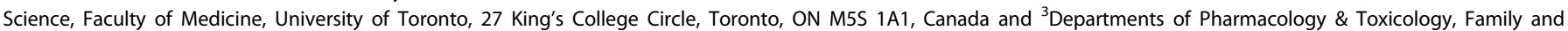
Community Medicine and Psychiatry, University of Toronto, 27 King's College Circle, Toronto, ON M5S3H7, Canada

Correspondence: Bernard Le Foll (Bernard.lefoll@camh.ca)
}

Published online: 11 September 2019 


\section{ADDITIONAL INFORMATION}

Publisher's note Springer Nature remains neutral with regard to jurisdictional claims in published maps and institutional affiliations.

\section{REFERENCES}

1. Elsaid S, Kloiber S, Le Foll B. Effects of cannabidiol (CBD) in neuropsychiatric disorders: a review of pre-clinical and clinical findings. Prog Mol Biol Transitional Sci 2019 (accepted for publication).

2. Kaplan JS, Stella N, Catterall WA, Westenbroek RE. Cannabidiol attenuates seizures and social deficits in a mouse model of Dravet syndrome. Proc Natl Acad Sci USA. 2017;114:11229-11234.
3. Devinsky O, Patel AD, Cross HJ, Vicente V, Wirrell EC, Privitera M, et al. Effect of cannabidiol on drop seizures in the Lennox-Gastaut syndrome. New Engl J Med 2018;378:1888-1897.

4. Bakas T, van Nieuwenhuijzen PS, Devenish SO, McGregor IS, Arnold JC, Chebib M. Pharmacol Res 2017;119:358-370.

5. Blessing EM, Steenkamp MM, Manzanares J, Marmar CR. Cannabidiol as a potential treatment for anxiety disorders. Neurotherapeutics 2015;12:825-836.

6. Elms L, Shannon S, Hughes S, Lewis N. Cannabidiol in the treatment of post-traumatic stress disorder: a case series. J Altern Complement Med 2019; 25:392-397.

\title{
OPEN
}

\section{Is depression a disorder of electrical brain networks?}

\author{
Yael Grossman ${ }^{1}$ and Kafui Dzirasa ${ }^{1,2}$ \\ Neuropsychopharmacology (2020) 45:230-231; https://doi.org/10.1038/s41386-019-0511-8
}

Major depressive disorder (MDD) is one of the most prevalent and disabling neuropsychological disorders in the world, with $15 \%$ of adults expected to experience depression sometime in their lives. Current treatment options are largely ineffective, as only $50-70 \%$ of patients experience remission after multiple rounds of treatment [1]. Thus, there is a clear and immediate need for the development of novel therapeutics that prevent MDD. Nevertheless, this endeavor has been hampered by limited knowledge of the biology underlying the disorder.

A well-validated murine model of depression, chronic social defeat stress (CSDS) [2], can differentiate between mice that exhibit MDD-like behavior following stress exposure, termed "susceptible", and those that do not, termed "resilient". Our lab's prior work exploring network dynamics linked to CSDS susceptibility found that susceptible mice exhibited greater prefrontal cortex (PFC)-dependent limbic synchrony [3]. Since susceptible and resilient mice experienced identical stress exposure but exhibited different network dynamics after CSDS, we hypothesized that differences in network dynamics exist prior to stress exposure and could serve as a biomarker for the vulnerable population of test mice (i.e., mice that will exhibit MDD-like behavior following future exposure to (SDS).

To test this hypothesis, multicircuit recordings during acute threat were collected from test mice before and after exposure to CSDS, and processed using discriminative cross-spectral factor analysis (dCSFA), a model of machine learning [4]. The dCSFA method was chosen for its interpretability (i.e., relatability to specific neural phenomena) and prediction (i.e., discrimination of behavioral variables). This approach identified four electrical network features, termed "electome factors". These networks were validated using techniques previously demonstrated to increase vulnerability (e.g., early life stress, inflammation, and overexpression of the gene $S d k 1$ in the ventral hippocampus). Only one of these electome factors,
Electome Factor 1 (EF1), was responsive to vulnerability manipulations and, consequently, validated as a network underlying vulnerability. Furthermore, techniques for treating susceptible mice after CSDS (e.g., ketamine administration and suppression of activity in PFC) did not have any significant effect on EF1, though these treatments suppressed other electomes associated with susceptibility. Activity in this network originates in the PFC and ventral striatum, relays through the amygdala and ventral tegmental area, and converges in the ventral hippocampus. Together, these results indicate that $E F 1$ is a biomarker of vulnerability and is distinct from MDD-like susceptibility.

Alternative techniques have identified networks indicative of individual vulnerability to social stress in rats [5]. Though vulnerability identification has not progressed to humans yet, recent functional magnetic resonance imaging studies in depressed patients have revealed distinct functional networks [6]. Furthermore, differences in functional connectivity successfully predict different subtypes of depression as well as responsiveness to treatment, suggesting that network-level analyses may provide an avenue for developing more successful treatments for depression.

Our findings demonstrate that network-level spatiotemporal dynamics can indicate previously obscured vulnerable individuals within heterogeneous populations. These results could support the development of novel therapeutic mechanisms targeted at preventing the emergence of MDD or encouraging resilience in vulnerable populations. Furthermore, they encourage exploration of electome networks that may signal other emotional states in health and disease.

\section{FUNDING AND DISCLOSURE}

NIH Grant R01MH120158 to K.D. The authors declare no competing interests.

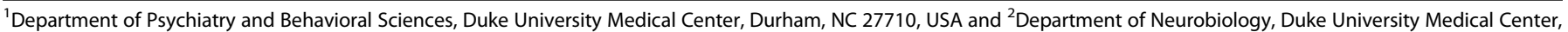
Durham, NC 27710, USA

Correspondence: Kafui Dzirasa (kafui.dzirasa@duke.edu)

Published online: 10 September 2019 